\title{
Надежность автомобильных ИС: экономические и технологические аспекты
}

\author{
М. Макушин ${ }^{1}$, В Мартынов, д. т.н., проф.²
}

УДК 621.38 | ВАК 05.27 .06

\begin{abstract}
Средства метрологии и тестирования относятся к основным методикам контроля надежности ИС, особенно автомобильных. В этом сегменте соответствующие требования намного жестче по сравнению с требованиями к ИС для потребительской техники. Для обеспечения надежности автомобильных ИС разрабатываются специальные программы непрерывного совершенствования и стратегии повышения качества, устанавливаются дополнительные требования к чувствительности управления технологическим процессом (необходимым для выявления потенциальных скрытых дефектов), методикам мониторинга отклонений по всему технологическому процессу завода - производителя автомобильных ИС, вводятся новые перспективные методы контроля дефектов на уровне кристалла. По мере развития рынка автомобильных ИС, обусловленного появлением и расширением парка подключенных (к сетям), полуавтономных и автономных транспортных средств, повышается актуальность вопросов надежности автомобильных ИС.
\end{abstract}

\section{ЭКОНОМИЧЕСКИЕ АСПЕКТЫ РАЗВИТИЯ ИНДУСТРИИ АВТОМОБИЛЬНЫХ ИС}

Еще несколько лет назад электроника рассматривалась преимущественно как часть потребительского информационно-развлекательного оборудования, а не как существенный функциональный компонент автомобиля. Кроме того, доля стоимости электронного содержимого автомобилей непрерывно повышается. По данным ICInsights, среднегодовые темпы роста продаж автомобильных интегральных схем в сложных процентах (CAGR) за 2016-2021 годы составят 13,4\%. В 2016 году продажи ИС увеличились на 11\%, а в 2017-м - на 22\% (до 28 млрд долл.). По итогам 2018 года объем рынка оценивается в 32,4 млрд долл., то есть на 16\% больше, чем в предыдущем году.

По данным исследовательской корпорации IHS Markit, ожидается, что в 2040 году общий объем продаж автономных транспортных средств в мире превысит отметку в 33 млн шт. Первые значительные поставки подобных машин (помимо транспортных средств, прошедших проверку на работоспособность) появятся на рынке в 2019 году. В 2021-м индивидуальным пользователям будет отгружено 51 тыс. шт. В 2025 году объем сбыта машин коллективного

ЦниИ «Электроника», главный специалист,

mmackushin@gmail.com

ФГБНУ «Аналитический Центр", проф., Минобрнауки РФ, эксперт. (такси) и индивидуального использования превысит 1 млн шт. Ключевое значение для серийного внедрения и развития новых технологий будут иметь комплексные услуги в рамках совершенствования систем услуг типа Uber, Lyft и т.д., связанных с обеспечением поездок по деловым и личным целям.

Одна из тенденций развития индустрии автономных транспортных средств в предстоящие десятилетия - существенное снижение количества дТП, в том числе со смертельным исходом. В связи с этим к инструментальным средствам тестирования автомобильной электроники и ИС, ориентированных на обеспечение безопасности транспортных услуг, предъявляются все более жесткие требования.

По некоторым оценкам, современный рынок оборудования автоматического тестирования автомобильных ИС составляет около 120 млн долл. в год. Однако есть и «размытые» позиции. Действительно, универсальные инструментальные средства тестирования микроконтроллеров способны проверять приборы для различных вариантов применения, включая автомобильную электронику. Но требования к надежности автомобильных систем повышенные [1]

\section{АКТУАЛЬНЫЕ ПРОБЛЕМЫ ТЕСТИРОВАНИЯ}

Возрастающая сложность конструкций ИС, масштабирование и экономические факторы все более затрудняют 
(если не делают невозможным) нахождение всех дефектов конструкции ис. Более того, тестирование становится не просто одним из завершающих этапов производства ИС, а неотъемлемой частью постоянно усложняющегося технологического процесса в целом, - компонентом, для которого характерен собственный сложный набор компромиссов.

Особенность текущей ситуации состоит в том, что современные ИС не могут быть протестированы с достаточной достоверностью на одной установке. Нужно изменять подходы (методики), причем по всей цепочке поставок, начиная от "проектирования под тестируемость" (DFT") на самых ранних этапах, необходимо также расширять область тестирования, то есть увеличивать количество контролируемых производственных операций.

Можно выделить три основные задачи, требующие решения:

- по мере масштабирования и повышения плотности размещения элементов большее количество участков ИС требуют проверки, что приводит к увеличению времени тестирования и использованию дополнительного оборудования;

- вокруг кристалла ИС располагается все больше разнородных элементов, масштабирование затрудняется, особенно в случаях таких приложений, как искусственный интеллект и облачные вычисления (а это значительно увеличивает стоимость программ тестирования в результате все большего числа взаимодействий, которые трудно планировать);

- в случае многокристальных решений отдельные кристаллы могут оказаться недоступными для средств тестирования, что увеличивает риски отказов (необходимую информацию можно получить только опытным путем в рамках методик разрушающего контроля).

Каждый из этих факторов увеличивает время тестирования, повышает сложность программ контроля и общую стоимость процесса тестирования. Как показывает опыт полупроводниковой промышленности, до сих пор затраты на тестирование составляли от 2 до 3\% общего бюджета проектирования ИС. Но по мере масштабирования затраты на операции тестирования возрастают.

Решение проблем тестирования требует значительных инноваций в области не только контрольно-испытательного оборудования, но и методологии, формирования новых стратегий тестирования. Предполагается, что частичным решением проблемы может стать повторное использование данных, полученных в ходе лабораторных

\footnotetext{
DFT (design for test (testability) - «проектирование под тестируемость», наименование методики проектирования, добавляющей специальные свойства тестируемости в процесс проектирования изделий микроэлектроники.
}

испытаний в допроизводственный период. По мере увеличения объема тестовых данных нужно рассматривать их во взаимосвязи с данными контроля всех компонентов, понимать, что происходит на уровне пластины на самых ранних этапах обработки. Один из популярных подходов - стратегия увеличения параллелизма тестирования, но и этого уже недостаточно. Наиболее желательная стратегия для проектировщиков - системное тестирование, включающее в себя тепловое, трехтемпературное (три диапазона температур для ИС военного, промышленного и коммерческого применения) и функциональные испытания. Это и означает массовое параллельное тестирование. Параллелизм увеличивает сложность, при этом дает больший объем данных контроля. И как следствие, усложнение процесса тестирования затрудняет перенос лабораторных разработок в реальное производство и повышает эффективность при освоении массового производства [2].

\section{ПРОБЛЕМЫ РАЗВИТИЯ АВТОМОБИЛЬНЫХ ИС}

Ожидаемое увеличение подключенных (к сетям) и автономных транспортных средств привлекает на рынок автомобильных ИС новых поставщиков. Подобные транспортные средства - предельно сложные системы, содержащие новейшую цифровую логику, различные типы процессоров и ускорителей, непростые датчики. Практически все эти технологические решения взаимосвязаны, что обусловлено набором законодательных и регулятивных требований, которые, в частности, закрепляют порядок и методы тестирования автомобильной электроники и ИС. Соответственно, процесс тестирования продолжает усложняться.

Для поставщиков ИС разработка приборов для автомобильной электроники сопряжена с рядом трудностей. Кавтомобильным ИС предъявляются жесткие требования по обеспечению безопасности, их тестирование должно быть более тщательным по сравнению с приборами для Rack-серверов или ИС для смартфонов. Это относится к формирователям сигналов изображения автомобильных камер, лидарам, радарам, а также микроконтроллерам, аналоговым ИС и ИС управления режимом электропотребления. Требуются как более точное определение функциональности, так и комплексное проектирование на системном уровне.

Все это предполагает наличие большего времени и усилий, при этом производители автомобилей настаивают на сдерживании роста издержек. Распространено мнение, что стоимость электроники в транспортных средствах бюджетного класса не должна выходить за пределы 2,0-5,0 тыс. долл.

Ранее для других вариантов применения (не для транспортных средств) полупроводниковая промышленность могла снижать стоимость решений путем уменьшения 
неповторяющихся (разовых) расходов на проектирование и внедрение в производство (NRE), сокращения издержек собственно производства и корпусирования благодаря интеграции в единую систему. Однако для автомобильной электроники такая модель уменьшения стоимости не всегда подходит. Ежегодно в мире продается примерно 90 млн легковых автомобилей и 2 млрд смартфонов. Если пользователь может менять смартфон каждый год, то в случае с машиной это маловероятно.

Еще один важный фактор состоит в том, что количество испытаний (а также этапов верификации конструкций ИС, модулей и плат) для автомобильных ИС значительно больше, чем для ИС, предназначенных для других областей применения, что тоже связано с обеспечением максимальной безопасности. Поэтому единственный способ существенного сокращения издержек на тестирование автомобильных ИС - оптимизация издержек по всей цепочке поставок. Один из возможных путей сокращения издержек - тестирование системного уровня.

Методика тестирования системного уровня уже используется в отношении узлов, состоящих из большого числа компонентов и реализованных по наиболее передовым технологическим нормам. Действительно, чем меньше топологические нормы, тем больше число транзисторов на кристалле. Соответственно, тестирование системного уровня дает преимущества. Встроенные аппаратные и/или программные средства самотестирования (BiST) теоретически позволяют обеспечить почти 99,5\%-ный охват, но в случае тестирования миллионов транзисторов этот показатель не превышает 85\%. Например, на уровне 10-нм топологий около 33\% времени процесса контроля затрачивается на подачу к испытуемому участку рабочего напряжения 100 мВ.

Приходится учитывать, что современные автомобили представляют собой сложные “системы систем», причем нельзя исключать взаимовлияния отдельных систем, особенно, когда некоторые из них более современные. Поэтому одна из стратегий поставщиков автомобильных ИС и электронных систем - использование новейших технологических процессов, чтобы максимально увеличить срок службы конструкций приборов / схем. Это особенно актуально для ИС, предназначенных для информационно-развлекательных систем и искусственного интеллекта, где от использования новейших топологий зависит повышение производительности, снижение потребляемой мощности и темпов морального устаревания.

Тенденция развития автомобильных ИС и электроники ведет к созданию архитектур, превращающих автомобиль в "суперкомпьютер". Архитектуры, объединяющие камеры или средства формирования изображения, обеспечивают водителю максимальную осведомленность об окружающей автомобиль среде. Подобное наращивание возможностей автомобильных систем сопряжено с расширением различных видов тестирования. В будущем возможно появление тестов системного уровня, встроенных в системы автомобиля, автомобильную электронику и отдельные ИС. При этом будет возможность подключаться к внешней среде тестирования, что имеет определенные преимущества.

Во-первых, при неожиданном выявлении новых угроз / проблем отказа транспортного средства (особенно в случае, когда до стационарного автосервиса далеко) возможность протестировать автосистемы / блоки дистанционно по беспроводным сетям (подключиться к внешней среде тестирования) позволяет проанализировать возникшую угрозу и решить, насколько срочно надо отзывать машину для исправления дефектов.

Во-вторых, возможность подключения бортовых средств тестирования к внешним средам тестирования в стационарных условиях позволит ускорить процесс тестирования, и в некоторых случаях полнее, чем в беспроводном режиме, модернизировать встроенные системы тестирования, а также проверить сами эти встроенные системы.

\section{КОНТЕКСТНОЕ ТЕСТИРОВАНИЕ}

Тестирование, обусловленное контекстом, или контекстное тестирование, - один из новых подходов, впервые появившихся в сфере разработки ПО, направленных на повышение эффективности процесса, более глубокое понимание тестовых данных, выявление ошибок и т. п. Производители автомобилей требуют 100\%-ного тестирования комплектующих, но это дорого и осложняется тем, что поставщики компонентов не обмениваются данными по всей цепочке поставок.

Если современные методики тестирования автомобильных ИС и электроники основаны на получении данных, то на следующем этапе предполагается использовать технологии больших данных. При этом возникает вопрос: будет ли ПО коррелировать данные с учетом выявляемых тенденций?

Эта одна из самых больших перемен в мире тестирования относится не только к оборудованию, но и к развитию автомобильной промышленности. Через 5-10 лет, когда автономные автомобили получат широкое распространение, обеспечение безопасности ПО автомобильной электроники может стать важнее безопасности отдельных компонентов и автомобильных ИС.

Автомобильные технологии и стандарты постоянно изменяются. Продолжают развиваться перспективные системы помощи водителю (ADAS) и технологии автоматизированного вождения, при этом общая тенденция - преобразование первых во вторые (рис. 1). Вся экосистема находится в движении - фирмам нужно понять, что создавать самостоятельно, а что отдавать на аутсорсинг. В этих условиях важный фактор - возможность 


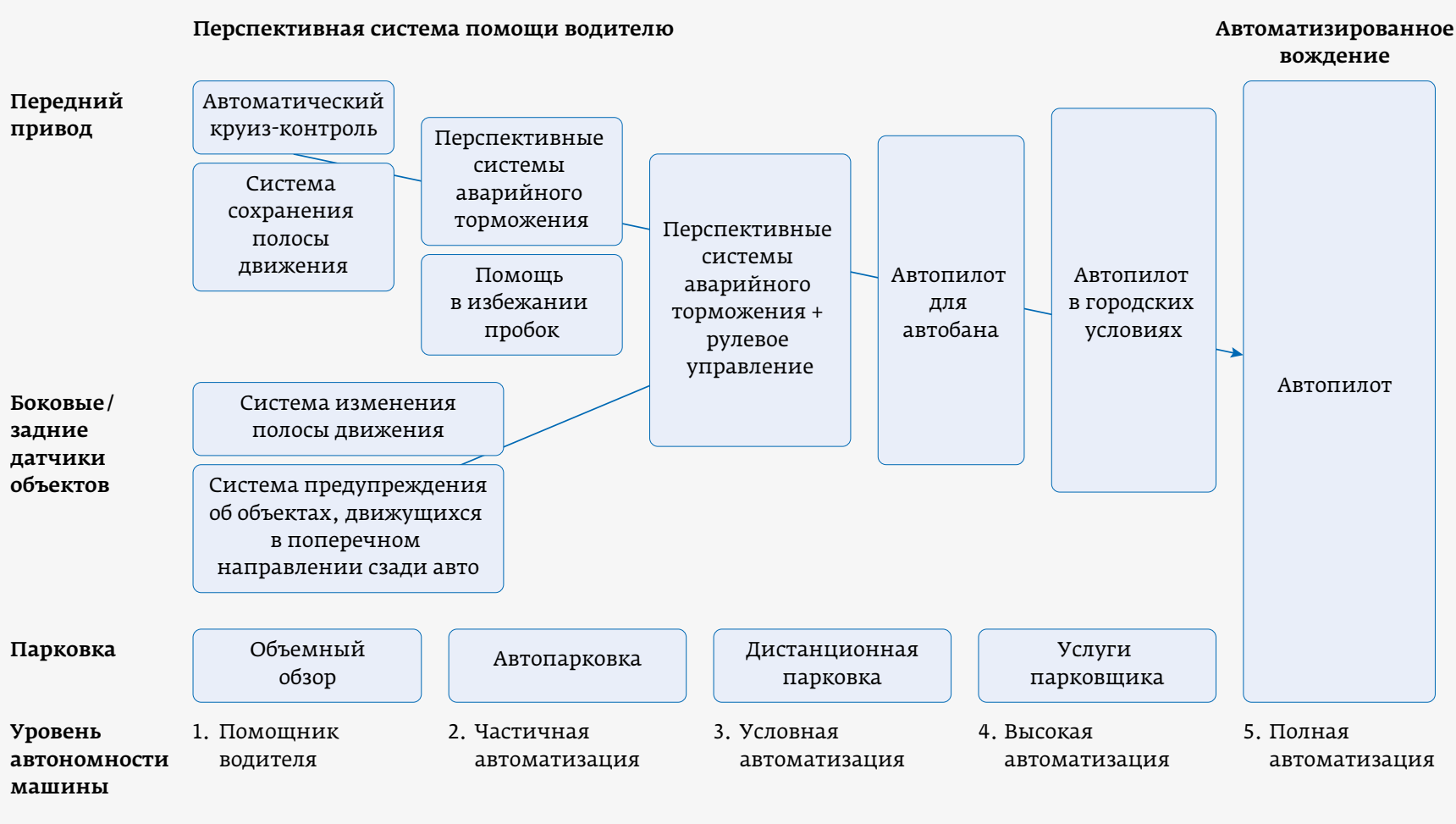

Рис. 1. Тенденция развития - от систем помощи водителю к автоматизированному вождению

поддержания и повышения качества всей расширяющейся цепи поставок.

Ранее в автомобильной промышленности применялись небольшие микроконтроллеры и другие приборы, изготавливаемые по отработанным, надежным процессам. Соответственно, качество и надежность поддерживать было достаточно легко. При переходе кавтономным транспортным средствам значительно усложняются приборы и системы, обеспечивающие функционирование ADAS и перспективных сетей управления автомобильным движением. Соответственно, тестирование автомобильных ИС во все большей мере будет ориентироваться на средства встраиваемого тестирования и тестирования системного уровня в контексте всех используемых в автомобиле компонентов, узлов и систем. Обращают на себя внимание псевдослучайные модели, модели цепей сканирования, а также совершенствование моделей тестирования по принципу «годен / не годен».

В сегменте автомобильных радаров для автомобилей премиум-класса основная тенденция состоит в снижении стоимости изделий в целях превращения их в стандартное оборудование для всех классов автомобилей. Для этого предстоит решить ряд задач, включая методики тестирования, дефицит специалистов в области Свч-техники, освоение 5G-технологии (в Т. ч. для анализа данных автономных транспортных средств, не требующих ответа в реальном масштабе времени) и т. п. [1].

\section{ДЕФЕКТНОСТЬ И ЦЕПЬ ПОСТАВОК: ЭКОНОМИЧЕСКИЕ И ТЕХНОЛОГИЧЕСКИЕ ФАКТОРЫ}

В 1950-е годы доля электроники в общей стоимости автомобиля составляла менее 1\%. В настоящее время этот показатель превышает 35\%, а к 2030 году увеличится до 50\% [3]. Быстрый рост спроса на электронику в автомобильной промышленности наблюдается по четырем основным направлениям:

- системы мониторинга и управления, электронная подача топлива, управление газоэлектрическими гибридными автомобилями и т. п.;

- безопасность (антиблокировочная система, подушки безопасности и т.д.);

- перспективные системы помощи водителю (ADAS) (предупреждение об уходе с выбранной полосы движения, помощь при парковке, мониторинг слепых зон, адаптивный круиз-контроль и т. п.);

- средства повышения комфортности (спутниковая навигация, информационно-развлекательное оборудование и т.д.) [4].

Ежегодно в мире производится более 88 млн автомобилей и легких грузовиков, каждому из которых требуются тысячи ИС. Отказ любой ИС в условиях эксплуатации может привести не только к необходимости гарантийного ремонта, но и к травмам или даже гибели водителя и пассажиров. 
Допустим, что средний автомобиль содержит 5 тыс. интегральных схем, изготовитель производит 25 тыс. автомобилей ежедневно. При уровне отказов 1: 1000000 это означает, что более 125 автомобилей каждый день испытывают проблемы с надежностью [5]. Поэтому производители систем автомобильной электроники в соответствии с требованиями автопроизводителей стремятся к уровню надежности с частотой отказов 1:1000 000000. Современные методы обнаружения отказов, влияющих на надежность, в большой мере зависят от способов тестирования и испытаний на отказ, в результате целевые показатели качества могут отклоняться от реальных почти на порядок. Наиболее простым и низкозатратным является контроль качества ИС на заводе-изготовителе, который располагает оборудованием и возможностью исправления некоторых дефектов. Соответственно, для выхода на растущий рынок автомобильной электроники (или для сохранения своей доли продаж) изготовителям ИС необходимо совершенствовать способы повышения надежности их продукции.

Однако надежность микросхем в высокой степени коррелирует со случайной дефектностью. На раннем этапе жизненного цикла ИС при условии хорошо отработанного процесса и конструкции преобладают случайные дефекты. Катастрофический дефект (воздействующий на выход годных) приводит к отказу устройства в момент $\mathrm{t}=0$ (завершающее тестирование). Скрытый дефект (влияющий на надежность) становится причиной отказа в момент $t>0$ (при испытании на отказ). Взаимосвязанные катастрофические и скрытые дефекты оказывают влияние на выход годных и на надежность. Два этих типа дефектов различаются по размерам и месту возникновения в приборной структуре. На рис. 2 показаны примеры катастрофических и скрытых дефектов, приводящих к размыканию цепи и короткому замыканию.

Взаимосвязь дефектов, влияющих на выход годных и надежность, не ограничивается несколькими типами конкретных неполадок. Любой дефект, способный привести к снижению выхода годных, потенциальная угроза надежности. Анализ отказов показывает, что большая часть дефектов, влияющих на надежность, на самом деле связана с технологическими производственными процессами. Поскольку у дефектов, воздействующих на выход годных и надежность, одна и та же первопричина, увеличение выхода годных (за счет снижения воздействующих на него дефектов) будет приносить дополнительные выгоды в плане повышения надежности. Оптимальный способ снизить вероятность появления скрытых дефектов - снижение общего уровня случайной дефектности, что, в свою очередь, подразумевает:

- обеспечение более высокого уровня выхода годных;

- снижение частоты отклонений от стандартных параметров технологического процесса;

- быстрое обнаружение и исправление отклонений от параметров технологического процесса в случае их возникновения;

- отбраковку подозрительных кристаллов с использованием контроля на уровне кристалла [4].

Устойчивая связь между выходом годных и надежностью полупроводниковых ИС достаточно хорошо изучена и задокументирована, что отражено на рис. 3. Подобные результаты были показаны на уровне кристалла, пластины и партии пластин. Условно говоря, высокий выход годных обеспечивает высокую надежность. Подобная корреляция выхода годных ожидаема, так как типы дефектов, ведущих котказам кристалла, практически те же, что и вызывающие проблемы надежности на начальном этапе эксплуатации. Различаются дефекты выхода годных и надежности в первую очередь размерами и местом возникновения в рисунке на кристалле ИС.

По мере уменьшения числа катастрофических дефектов выхода годных в процессе производства ИС повышается базовый уровень выхода годныхи одновременно надежность прибора при эксплуатации. Соответственно, возникают два вопроса:

- каков уровень затрат времени, денег и ресурсов для достижения необходимой надежности?

- какие методики снижения дефектов и повышения базового уровня выхода годных являются оптимальными для достижения требуемой надежности?

Для заводов, изготавливающих ИС для потребительской электроники (мобильных телефонов, планшетных ПК и т.д.), «зрелый выход годных» (выход годных освоенного и отработанного в условиях массового производства технологического процесса) определяется как точка, где

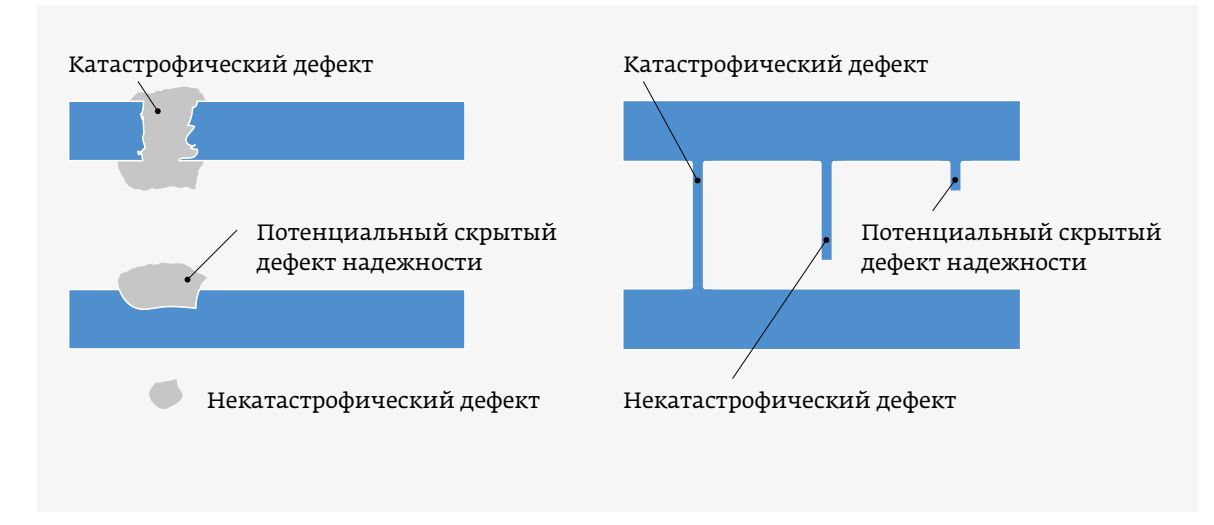

Рис. 2. Типы дефектов, влияющих на выход годных и надежность 


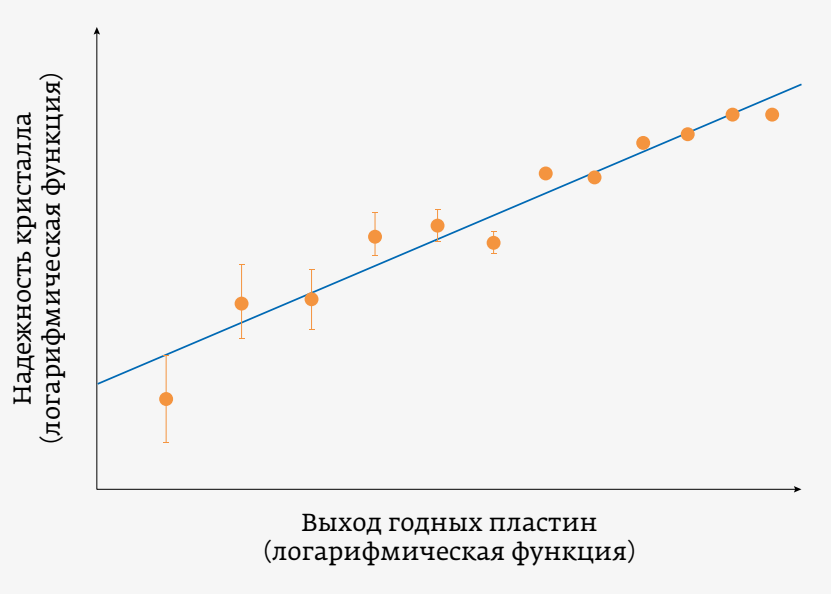

Рис. 3. Устойчивая корреляция между выходом годных и надежностью полупроводниковых ИС

дальнейший рост выхода годных не гарантируется вложениями времени и ресурсов. Как только изделие достигает зрелости, выход годных имеет тенденцию стабилизироваться на высоком значении, которое, однако, значительно ниже 100\%. Для заводов по обработке пластин для бытовой электроники больший экономический смысл не в попытках достичь более высокого уровня выхода годных, а в перераспределении ресурсов на разработку приборов и процессов следующего технологического поколения или на снижение издержек в целях повышения рентабельности продукции.

Для производителей автомобильных ИС экономическое решение об увеличении инвестиций в повышение выхода годных выходит за пределы типичного определения предельного дохода. В случае проблем с надежностью производитель автомобильных ИС, скорее всего, будет нести расходы на дорогостоящий и трудоемкий анализ отказов, а также на повышение ответственности за гарантийные сбои при эксплуатации, отзывы (изделий) и потенциальную правовую ответственность. Поскольку требования к надежности автомобильных ИС на два-три порядка превышают требования к потребительским ИС, поставщики автомобильных ИС должны обеспечивать более высокие базовые уровни выхода годных. Это требует нового образа мышления в плане того, что представляет собой «зрелый выход годных».

На рис. 4 показано различие «зрелого выхода годных» у изготовителей потребительских и автомобильных ИС. Поскольку выход годных постоянно увеличивается, можно сделать вывод, что почти все систематические причины снижения выхода годных были устранены у изготовителей как потребительских, так и автомобильных ИС. Наблюдаемое снижение выхода годных (см. рис. 4, закрашенная область) возникает в основном из-за случайных дефектов, привнесенных использовавшимися в процессе

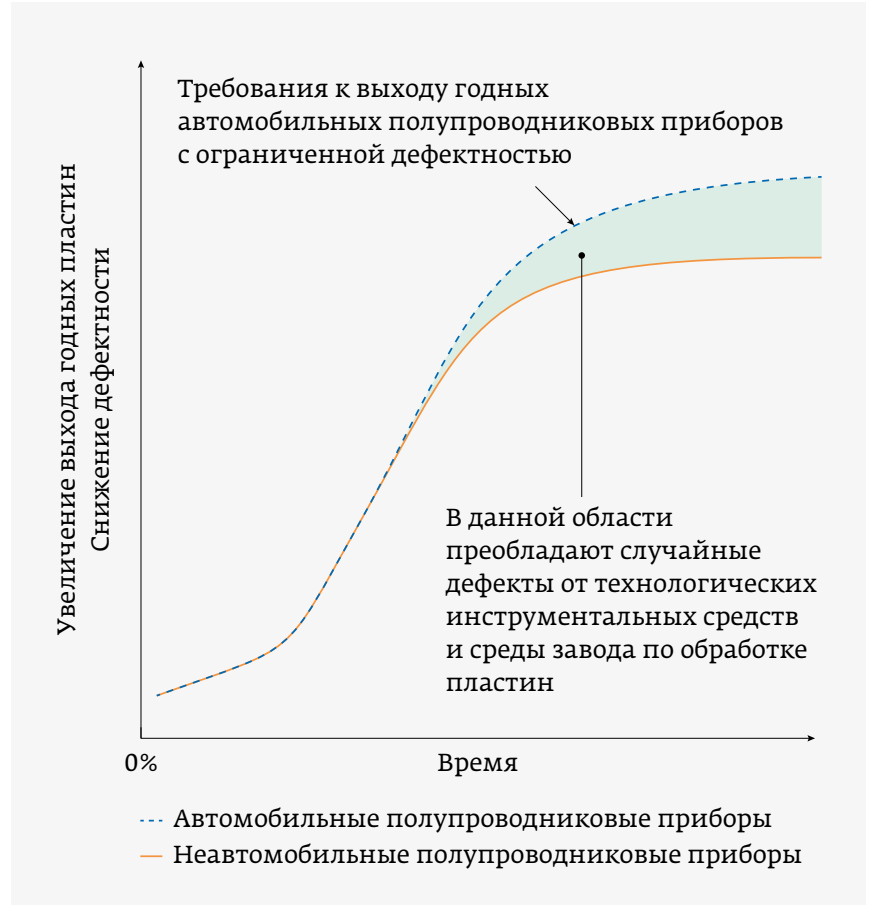

Рис. 4. Сопоставление роста выхода годных заводов по производству автомобильных и неавтомобильных полупроводниковых приборов

производства инструментальными средствами либо окружающей средой. Если производитель потребительскихиС может оценить выход годных и надежность по принципу "достаточно хорошо", то производители автомобильных ИС должны постоянно совершенствовать производственные процессы, чтобы уменьшить количество дефектов, увеличить выход годных (см. рис. 4). За счет уменьшения числа ограничивающих выход годных дефектов они также снижают количество скрытых дефектов надежности, оптимизируя прибыли и снижая риски.

В цепи поставок автомобильной промышленности - от изготовителей комплектного оборудования (OЕM) до поставщиков первого уровня и производителей ИС - «значение имеет каждый дефект», поэтому они придерживаются стратегии «Нулевая дефектность». После отгрузки ИС с завода затраты на обнаружение ИС с дефектами и смягчение последствий увеличиваются десятикратно на каждом дополнительном уровне цепи поставок. Таким образом, чрезмерное доверие к электрическим испытаниям следует заменить стратегией, обеспечивающей выявление скрытых отказов у производителя ИС, где стоимость соответствующих работ наиболее низкая. Продвижение завода к "Нулевой дефектности» возможно только путем реализации систематической программы снижения уровня дефектов. Достижение этой цели позволит изготовителям ИС пройти строгие проверки, устраиваемые автопроизводителями. 
В дополнение к надежным встраиваемым (в производственный процесс) возможностям контроля дефектов существует ряд методик снижения дефектов, на которые полагаются специалисты автопромышленности по закупкам. Кним, в частности, относятся:

- программа непрерывного улучшения (Continuous Improvement Program, CIP) показателей дефектности на базовом уровне;

- технологический процесс Golden Tool (Golden Tool Work Flow);

- программы типа Dog Tool.

\section{НЕПРЕРЫВНОЕ УЛУЧШЕНИЕ ПОКАЗАТЕЛЕЙ ДЕФЕКТНОСТИ НА БАЗОВОМ УРОВНЕ}

Основа любого строгого плана снижения дефектов базового уровня - стратегия встроенного (в технологический процесс) обнаружения дефектов. Подобная стратегия обнаружения дефектов, воздействующих на выход годных и надежность по проектным нормам и типам приборов, должна включать в себя соответствующий набор инструментальных средств управления технологическим процессом и адекватный план выборочного контроля. Используемые системы контроля дефектов должны обеспечивать необходимую чувствительность, соответствовать спецификациям и применять хорошо отлаженные методы контроля. План выборочного контроля должен формироваться в соответствии с установленной последовательностью этапов технологического процесса и обеспечивать достаточно частую контрольную выборку или применение инструментальных средств. Кроме того, необходимо обеспечить удовлетворительные возможности контроля дефектов, позволяющие ускорить обнаружение проблем, изоляциюпервопричин и отслеживание разрабатываемого проекта (конструкции - work-in-progress, WIP) в зоне риска. Применение всех этих элементов микроэлектронным предприятием, выпускающим автомобильные ИС, даст возможность реализовать план снижения дефектов базового уровня. Со временем это обеспечит положительные тенденции в области выхода годных, позволит сформулировать цели совершенствования и достичь уровня лучших примеров отраслевой практики.

В рамках реализации плана снижения дефектности на базовом уровне одной из серьезнейших проблем остается обнаружение источника дефекта. Иногда дефект выявляется по завершении многих этапов процесса, последовавших после этапа, ставшего источником дефекта, и, например, сделавшихего более заметным для систем контроля. Стратегия мониторинга инструментальных средств помогает решить вопрос относительно происхождения дефекта.

В рамках приложений "Мониторинг инструментального средства/аттестация инструментального средства» (Tool Monitoring/Tool Qualification, TMTQ) контролируется необработанная пластина, ее прогон через специализированный технологический инструмент (или камеру), а затем осуществляется повторный контроль (рис. 5). Любые дефекты, обнаруженные на пластине при повторном контроле, добавляются этим технологическим инструментом. В подобном случае не приходится сомневаться в происхождении дефекта. Производители автомобильных ИС, соблюдающие стандарт нулевой дефектности, осознают преимущества стратегии контроля инструментальных средств: благодаря чувствительным способам контроля, надлежащим ограничениям контроля и планам действий вне сферы контроля можно выявить и рассмотреть источники случайных потерь выхода годных, внесенных каждым технологическим инструментом.

Составленный временной графиквлияния технологического инструмента на число добавленных дефектов можно использовать как иллюстрацию непрерывного повышения уровня дефектности, а также применять при дальнейших работах в этом направлении. Дефекты, создаваемые каждым инструментальным средством, используемым на заводе, можно классифицировать для создания библиотеки дефектов, которую можно использовать для анализа дефектов изделий, возвращаемых из эксплуатации. Сопоставление уровня привносимой дефектности двух технологических инструментов может показать, какой из них чище. Это поможет создать более точный график их технического обслуживания. Такой подход требует достаточно частой аттестации инструментальных средств-по крайней мере, раз в день - и обычно используется с технологическим процессом Golden Tool и программами Dog Tool.

\section{TЕХНОЛОГИЧЕСКИЙ ПРОЦЕСС GOLDEN TOOL}

Другая стратегия, используемая заводом по обработкепластин для достижения требований стандарта "Нулевая дефектность» в соответствии с запросами автомобильной промышленности, - технологический процесс Golden Tool. В рамках этого процесса (или Автомобильного технологического процесса - Automotive Work Flow, AWF) пластины для изготовления автомобильных ИС проходят только через наиболее совершенные технологические инструменты (golden tools) завода по обработке пластин. Поэтому заводские специалисты должны знать лучшие технологические инструменты для каждого этапа процесса. Для выявления оптимального технологического инструмента используются данные со встроенных средств контроля и инструментальных средств мониторинга, только потом выбранные технологические инструменты применяются в рамках AWF. Ограничение "автомобильных" пластин обработкой на одном технологическом средстве на каждом этапе технологического процесса может привести к увеличению времени цикла (обработки). Однако это обычно предпочтительнее обработки пластин, предназначенных для изготовления автомобильных ИС, с использованием технологических инструментов, характеризующихся более высоким уровнем 


\section{Инструментальные средства мониторинга}

Дефекты, "добавленные" данным инструментальным средством

Постконтроль

необработанной пластины

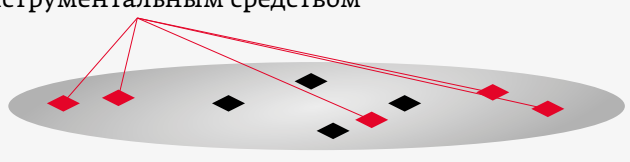

Пластина, прошедшая цикл технологического инструмента

Предварительный контроль необработанной пластины
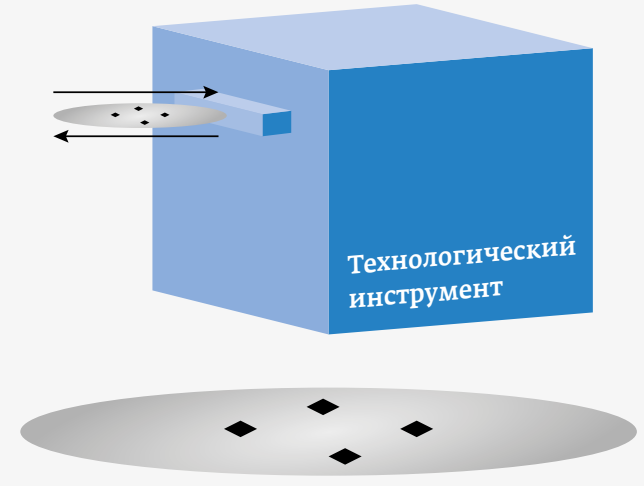

Tool требует наличия у завода систематической стратегии мониторинга инструментальных средств для аттестации каждого технологического инструмента на каждом этапе технологического процесса. Эта аттестационная процедура должна проводиться как минимум ежедневно по каждому инструменту для того, чтобы обеспечить наличие достаточного объема данных. Данные, используемые при анализе методами ANOVA или КрускалаУоллиса, помогут определить лучшие и худшие инструментальные средства в каждом комплекте. Программа Dog Tool с запланированным простоем технологических инструментов - один из самых быстрых (из известных) способов довести производство завода до уровня требований автомобильных стандартов. Данная стратегия, способствующая повышению выхода годных и надежности, в конечном счете, увеличивает эффективную мощность и рентабельность завода по производ-

Рис. 5. После предварительного контроля необработанная пластина может пройти через некоторые или все технологические инструменты. Постконтроль выявляет дефекты, добавленные технологическим инструментом

дефектности, что приводит к проблемам с надежностью ИС. В сочетании такого подхода с систематической программой непрерывного улучшения (уровня дефектности) большинство поставщиков ИС могут получить для каждого этапа технологического процесса несколько инструментальных средств, аттестованных по требованиям AWF.

Так как метод восходящего масштабирования достаточно труден, технологический процесс Golden Tool лучше подходит для заводов, где только малая доля разрабатываемых ИС предназначена для автомобильной промышленности. Для заводов с массовым производством автомобильных полупроводниковых приборов предпочтительнее использовать более системные программы непрерывного снижения уровня дефектности, такие как подход Dog Tool.

\section{ПРОГРАММA DOG TOOL}

Программа Dog Tool - противоположность технологическому процессу Golden Tool, поскольку она активно обращается ктехнологическим инструментам с низкими характеристиками (dog tools). Заводы, успешно снижающие дефектность базового уровня, зачастую использовали именно программы Dog Tool. Сначала Dog Tool выводится из производственной линии и совершенствуется до тех пор, пока его (инструмента) характеристики не станут лучше, чем средний показатель остальных инструментов данного комплекта. Эта операция повторяется до тех пор, пока все технологические инструменты комплекта не будут соответствовать минимальному стандарту. Эффективная программа Dog ству автомобильных ИС [6].

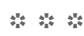

В статье не рассматривались вопросы стратегий мониторинга отклонений по всему технологическому процессу завода-производителя автомобильных ИС, обеспечивающих быстрое нахождение материалов с недопустимыми отклонениями, перспективные методы контроля дефектов на уровне кристалла. Об этом речь пойдет в дальнейшем, когда ведущие специалисты KLA-Tencor завершат серию статей, публикуемых в журнале Solid State Technology с января 2018 года

\section{ЛИТЕРАТУРА}

1. Dorsch J., Sperling E. Auto Chip Test Issues Grow // Semiconductor Emgineering. 2018. January 8.

2. Dorsch J., Sperling E. The Race To Zero Defects // Semiconductor Engineering. 2018. September 10.

3. Automotive electronics cost as a percentage of total car cost worldwide from 1950 to 2030 // Statista 2018.

4. Price D., Sutherland D., Rathert J. Process Watch: The (automotive) problem with semiconductors // Solid State Technology. Wafer News. 2018. January 15.

5. Cars That Go the Distance: Consumer Reports Car Reliability Survey 2017 Patrick Olsen // Consumer Reports. 2017. October 19

6. Price D., Sutherland D., Rathert J. Process Watch: Baseline yield pre-dicts baseline reliability // Solid State Technology. The Pulse. 2018. March 